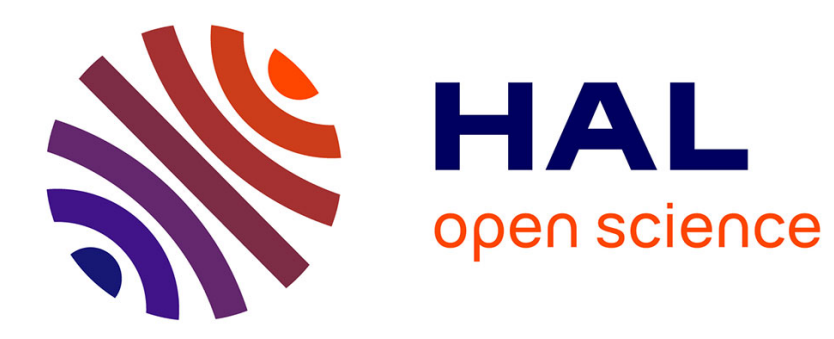

\title{
Psychologisation et dé-psychologisation de l'accompagnement des chômeurs
}

\author{
Sophie Divay
}

\section{To cite this version:}

Sophie Divay. Psychologisation et dé-psychologisation de l'accompagnement des chômeurs. Sociologies pratiques, 2008, 17, pp.55-66. halshs-00340009

\section{HAL Id: halshs-00340009 \\ https://shs.hal.science/halshs-00340009}

Submitted on 19 Nov 2009

HAL is a multi-disciplinary open access archive for the deposit and dissemination of scientific research documents, whether they are published or not. The documents may come from teaching and research institutions in France or abroad, or from public or private research centers.
L'archive ouverte pluridisciplinaire HAL, est destinée au dépôt et à la diffusion de documents scientifiques de niveau recherche, publiés ou non, émanant des établissements d'enseignement et de recherche français ou étrangers, des laboratoires publics ou privés. 


\title{
Psychologisation et dé-psychologisation de l'accompagnement des chômeurs
}

\author{
Sophie Divay ${ }^{1}$
}

\begin{abstract}
L'accompagnement des chômeurs a supplanté l'aide à la recherche d'emploi. S'agit-il d'une simple variation terminologique, d'un effet de mode ou d'un glissement sémantique chargé de sens? L'analyse des pratiques des intervenants du domaine de l'insertion professionnelle apporte quelques éléments de réponse à cette question. L'activité de conseil de ces professionnels semble en fait sous-tendue par un double mouvement de "dépsychologisation" et de rationalisation qui se produit sous l'influence de nouvelles orientations nationales et internationales de la politique de l'emploi.
\end{abstract}

\begin{abstract}
"Accompaniment" of unemployed people has replaced support of job seekers. Is it a simple terminological variation, a fashion trend effect or a terminological shift in meaning ? The analysis of professionals' work practices in the field of social welfare for the unemployed brings in light some responses. The activity of these professionals is actually challenged by a double trend movement of "de-psychologisation" and rationalisation due to the influence of new national and international unemployed policies.
\end{abstract}

Avec la progressive installation du chômage de masse, l'aide à la recherche d'emploi s'est au fil du temps développée et diversifiée. Cette forme de traitement social des chômeurs est à l'origine de la création de nombreux emplois d'intervenants plus ou moins proches du travail social. Ils peuvent, plus exactement, être rangés dans la nébuleuse des « nouveaux métiers de l'intervention sociale » (Ion, Ravon, 2005). Cette sphère a émergé au début des années 1980, parallèlement à la mise en place de politiques de l'emploi et de la ville, et en réponse aux difficultés économiques qui sont apparues à cette époque.

A ses débuts, l'aide à la recherche d'emploi ne faisait pas l'objet d'un intérêt particulier. Cette activité paraissait bien anodine et était perçue comme un soutien moral et une aide technique (conseils pour la rédaction de $\mathrm{CV}$, lettres de candidature, etc.) apportés aux chômeurs que l'on épaulait dans leurs démarches. Rien de bien intéressant finalement, un simple service rendu à des personnes plus ou moins en difficulté. Toutefois, en y regardant de plus près, on mettra tout d'abord en évidence que ces conseils contiennent des messages implicites et que les conseillers ont eu à adopter une approche psychologisante pour agir sur le comportement des chômeurs

Vers la fin des années 1990, les politiques de l'emploi internationales font sortir de l'ombre l'aide à la recherche d'emploi, notamment avec la mise en avant du principe d'activation des chômeurs et l'injonction du "retour rapide à l'emploi ». Comme on le verra, dans un deuxième temps, ces orientations internationales entraînent une redéfinition de l'aide à la recherche d'emploi qui s'intensifie et se généralise notamment en France.

\footnotetext{
${ }^{1}$ Centre d'Economie de la Sorbonne - Equipe Matisse
} 
Enfin, alors qu'il est de moins en moins question d'aide à la recherche d'emploi et de plus en plus d'accompagnement des chômeurs, on constate que la démarche des intervenants évolue. Ces derniers sont désormais soumis à de fortes exigences en termes de taux de placement de leurs publics et disposent, de ce fait, de moins de temps pour être à l'écoute de leurs interlocuteurs. Comme le dit une conseillère rencontrée, il faut être « dans l'opérationnel ». Une tendance à la «dé-psychologisation » s'amorce donc, notamment sous l'effet d'un processus de rationalisation qui a d'abord touché les chômeurs et qui depuis peu se manifeste à travers une formalisation accrue des pratiques des conseillers. Mais commençons, avant toute chose, par découvrir les caractéristiques de ce groupe professionnel aux contours imprécis.

\section{Encadré méthodologique}

Cet article s'appuie sur les résultats d'une recherche en cours portant sur « L'accompagnement vers et dans l'emploi », commanditée par la DARES, démarrée en novembre 2006 et impliquant des chercheurs sociologues et économistes de trois équipes (le GREE - Université de Nancy 2, le LESSOR - Université de Rennes 2 et le Matisse du Centre d'Economie de la Sorbonne).

Cette recherche a pour objectif d'analyser l'accompagnement des chômeurs à partir d'une synthèse raisonnée de la littérature et d'une recherche empirique à caractère monographique portant sur les pratiques d'accompagnement auprès de conseillers en aide à la recherche d'emploi relevant ou non du Service public de l'emploi.

Ont été utilisés ici les entretiens menés auprès de conseillers rattachés à des structures situées en Ile de France où le travail de terrain s'est effectué dans onze structures (une entreprise de travail temporaire, une association spécialisée dans l'aide à la recherche d'emploi des jeunes diplômés, un organisme de formation public, deux agences locales pour l'emploi, la Direction générale de l'Anpe, un "Cap vers l'entreprise ", une Maison de l'emploi municipale, deux Missions locales, un PLIE $^{2}$, un organisme de formation privé et une structure universitaire spécialisée dans l'insertion professionnelle des étudiants), soit auprès de 14 responsables au niveau régional ou local et auprès de 18 conseillers en aide à la recherche d'emploi.

\section{1- Les professionnels de l'aide à la recherche d'emploi}

Le champ de l'aide à la recherche d'emploi ne se laisse pas appréhender facilement tant il est dense, c'est-à-dire composé d'un très grand nombre de professionnels rattachés à des organismes à vocations variées, dont il n'existe pas d'inventaire précis et chiffré. Ils peuvent toutefois être classés dans quelques grandes familles distinctes.

En tout premier lieu, s'imposent, par leur rôle central dans le traitement du chômage, l'ensemble des agences locales de l'ANPE et l'AFPA qui incarnent le Service public de l'emploi (SPE) et qui sont, à ce titre, en charge de la mise en œuvre des politiques de l'emploi. Le panorama s'étoffe avec l'ajout, d'une part, des co-traitants de l'ANPE qui comprennent l'APEC ${ }^{3}$, les Missions locales et Cap Emploi ${ }^{4}$ et, d'autre part, des sous-traitants, dont le nombre s'élevait à 4300 en 2004 (Balmary, 2004). Pour clore le tableau très globalement, il faut encore signaler l'existence de structures sans lien avec le SPE. Elles interviennent auprès de personnes qui ne sont pas au chômage, mais qui vont être prochainement en recherche d'emploi. On compte, entre autres, les universités et leurs

\footnotetext{
${ }^{2}$ PLIE : Plan local pour l'insertion et l'emploi.

${ }^{3}$ APEC : Association pour l'emploi des cadres.

${ }^{4}$ Cap Emploi : Réseau de 119 organismes spécialisés auprès des personnes handicapées en démarche d'accès ou de retour à l'emploi en milieu ordinaire de travail.
} 
cellules d'insertion professionnelle, les grandes écoles, les cabinets privés de recrutement ou d'outplacement surtout au service des cadres supérieurs, les structures d'insertion par l'économique, etc. Cette liste incomplète laisse entrevoir la diversité des publics auprès desquels les conseillers interviennent, ainsi que la multiplicité de leurs employeurs potentiels. Ceci est sans doute une des raisons pour laquelle ces professionnels portent des titres d'emploi variés et en perpétuelle évolution. Par ailleurs, aucune formation spécifique n'est requise pour occuper ce type de poste, les employeurs exigeant plutôt un niveau d'études (autour de bac +4 ) ou une expérience dans l'insertion professionnelle.

Finalement, ce tableau peut donner l'impression d'un éclatement des profils des professionnels de l'aide à la recherche d'emploi. Leur homogénéité se trouve ailleurs. Elle réside principalement dans le partage d'un même rapport au travail et de pratiques semblables, fondées sur un même noyau de connaissances et de techniques, ainsi que dans la confrontation à des difficultés et contraintes semblables.

\section{2- L'aide à la recherche d'emploi : prédominance d'une interprétation psychologisante du social}

Les différentes recherches menées, à partir des années 1990, sur les pratiques des conseillers en aide à la recherche d'emploi ${ }^{5}$ mettent en évidence de nombreux points communs et notamment le recours à un mode d'intervention fortement psychologisant, même si tous les auteurs de ces travaux n'emploient pas explicitement ce terme.

Bien que des diplômes ou certificats soient depuis quelque temps délivrés dans le domaine de l'insertion professionnelle ${ }^{6}$, les intervenants n'ont en général pas appris à devenir des conseillers en aide à la recherche d'emploi dans le cadre d'une formation initiale. Les plus " outillés » sont sans doute les conseillers de l'ANPE, organisme qui a développé, dès le début des années 1980, de nombreuses méthodes d'intervention. Ces méthodes ont été reprises par les co-traitants ou sous-traitants de l'Agence, d'autant plus facilement qu'elles sont aujourd'hui accessibles sur Internet, sous la forme vulgarisée de petits guides d'abord destinés aux demandeurs d'emploi. Beaucoup d'organismes ont aussi créé leurs propres référentiels et méthodes sur la base de principes privilégiant l'orientation professionnelle, la médiation, ou le coaching, etc. D'autres se sont inspirés de théories et de modèles pédagogiques, telles que l' « activation du développement vocationnel et personnel » (ADVP) ou le « trèfle chanceux » conçu par un psychologue québécois.

Quoi qu'il en soit, les intervenants ont constitué leur propre «boîte à outils » et ceux qui ont occupé plusieurs postes de conseillers chez des employeurs différents disent qu'ils ont conservé les mêmes modes d'intervention. En effet, leur pratique s'est moins forgée sous l'influence de consignes hiérarchiques que dans la confrontation quotidienne à des situations, demandes, contraintes, difficultés et limites semblables. Bien que placés face à des publics en recherche d'emploi divers, les conseillers ont tous à traiter le même problème, celui de l'insertion professionnelle. L'observation montre, par exemple, que les techniques d'aide à la recherche d'emploi recouvrent, en dehors de quelques variantes, toujours les mêmes démarches : bilan personnel et professionnel, constitution d'un projet professionnel, rédaction d'un $\mathrm{CV}$, ciblage et enquêtes auprès des employeurs, lecture des petites annonces, rédaction

\footnotetext{
${ }^{5}$ Voir notamment : Beaud (1996), Béraud (2006), Castra (2003), Divay (1999, 2000, 2001), Frétigné (2004), Jellab (1997), Mauger (2001), Orianne (2005).

${ }^{6}$ Telle que la formation de « Conseiller en insertion professionnelle » de niveau III, dispensée par le CNAM.
} 
de réponses à ces annonces et de candidatures spontanées, simulation d'entretien d'embauche, de prises de contacts téléphoniques, analyse a posteriori des démarches effectuées, etc.

Aider un chômeur à trouver du travail, c'est avant tout améliorer son « employabilité » ou plus exactement sa « recrutabilité » (Pochic, 2007), néologisme qui met, comme le souligne son auteure, davantage l'accent sur les pratiques des employeurs que sur celles des demandeurs d'emploi. Cette tentative d'augmenter les chances d'embauche d'un chômeur recouvre entre autres un travail de socialisation ou de conformation (Macé, 1997) axé sur le comportement de l'individu qui doit apprendre à « se vendre », c'est-à-dire à mettre en valeur des qualités souvent morales (ponctualité, fiabilité, implication, etc.) auprès des recruteurs. Il doit notamment perfectionner ses compétences relationnelles pour incarner avec succès le rôle du candidat dans la scène de l'entretien d'embauche, et, pour ce faire, respecter les règles d'une interaction dissymétrique où il est en position dominée (Divay, 1999).

Selon les conseillers, l'acquisition de ces compétences nécessite une phase initiale de « travail sur soi », un temps de réflexion pendant lequel le chômeur dresse un bilan de son passé pour mieux définir un projet professionnel et personnel. Il lui faut alors passer par l'étape « connaissance de soi »; l'exercice classique consiste à se retrouver seul devant une feuille de papier comportant deux colonnes (les plus, les moins / les points forts, les points faibles / ce que j'aime, ce que je n'aime pas...) qui doivent l'aider à dégager les traits saillants de sa personnalité : « qui suis-je ? quelles sont mes envies? mes limites ? mes potentialités? »

La réussite de cette introspection dépend en grande partie du positionnement adopté par le conseiller. Il lui faut être à l'écoute, non directif, éviter tout jugement de valeur. Certains, de ce fait, vont momentanément jusqu'à perdre de vue le besoin premier de leur interlocuteur (trouver un emploi) pour se focaliser sur la verbalisation d'une «demande» ou plus simplement sur l'appropriation de la démarche normalisée du chercheur d'emploi.

Ce positionnement distancié obéit à un principe non interventionniste qui vise ce que Vrancken et Macquet (2006) nomment la « capacitation ou la capabilité des individus », c'està-dire la volonté d'autonomiser les chômeurs, de les rendre acteurs de leur parcours. Ils doivent se prendre en main, devenir responsables de leurs choix, de leurs réussites comme de leurs échecs.

Ce processus de responsabilisation, selon certains chercheurs en psychologie sociale (Beauvois et Le Poultier, 1985), recouvre une pression à l'internalité qui tend à renforcer l'attribution des conduites aux personnes, les conduisant à privilégier une forme de « contrôle interne » (c'est de ma faute) tout en éludant parallèlement le poids des structures sociales. L'occultation de l'environnement du chômeur est aisément adoptée par les conseillers qui n'ont que peu de prise sur le marché du travail et les exigences des employeurs.

L'aide à la recherche d'emploi consiste donc pour une grande part en un travail sur le comportement du chômeur qu'il s'agit de mobiliser ou (re)dynamiser, c'est-à-dire de maintenir dans un état de recherche active d'emploi. Faute de prise sur l'offre d'emploi (Castra, 2003), l'intervention se focalise sur l'individu et relève d'une approche psychologisante qui va de pair avec une décontextualisation, c'est-à-dire une occultation du marché du travail qui prévaut dans le cadre de ces « dispositifs centrés sur le travail sur soi, dans la perspective d'un emploi dont on ne dit pas, ou si peu, qu'il est une denrée rare. » (Benarrosh, 2000, p.25). 
La responsabilisation des victimes du chômage (ou comme dirait Goffman [1969], la tentative de «calmer le jobard ») connaît toutefois des limites. Elle risque, en effet, d'être contreproductive et de décourager les chômeurs, surtout les plus démunis ou les moins recrutables. Le conseiller doit ainsi maintenir un équilibre entre la stimulation plus ou moins culpabilisante et l'apport d'un soutien moral en offrant une écoute bienveillante :

" Il fallait qu'on soit là pour les rebooster, pour les pousser, pour leur redonner le courage de repartir, de pas lâcher prise, parce que c'est ça le gros souci avec les gens qui sont au RMI ... c'est-à-dire que ça fait quand même en général au moins deux ou trois ans qu'ils n'ont pas travaillé. Il y a des fissures, il y a des fêlures, et moi je leur ai toujours dit: 'si vous n'avez pas le moral, si vous sentez que ça va pas, vous m'appelez, vous me parlez de vos problèmes', parce que comme on pouvait pas leur proposer des choses vraiment pratiques, je trouvais que notre rôle c'était vraiment d'essayer de les rebooster, de leur redonner du courage. " (Conseillère d'une Maison de l'emploi municipale).

C'est ainsi que la psychologisation de l'aide à la recherche d'emploi est accentuée dans les situations de chômage de longue durée puisque, avec le temps, les individus risquent de plus de plus d'être marqués par les dommages de la précarité renforçant l'attention des intervenants sur leur personne et sur tous les problèmes à résoudre avant d'aborder la question de l'insertion professionnelle.

Cette grille de lecture psychologisante, qui sous-tend le mode d'intervention des conseillers, est depuis quelques années fortement remodelée sous l'influence de nouvelles orientations des politiques de l'emploi.

\section{3- Tournants dans le cours de l'histoire du traitement social des chômeurs}

Les politiques de l'emploi et les définitions du traitement social des chômeurs ont connu une nette évolution ces soixante dernières années qui s'est déroulée très schématiquement en trois grandes phases successives. La période d'après-guerre, allant de 1945 à 1973, est le théâtre de la mise en place des bases du Service Public de l'emploi actuel, dans le but d'organiser le marché du travail caractérisé par une pénurie de main-d'œuvre. Par la suite, un premier tournant se produit à la fin des années 1970 avec l'apparition d'un chômage structurel. Les mesures sont tout d'abord prises dans l'attente d'un retour à la normale, puis le constat d'une rupture économique s'impose et se traduit par l'instauration de programmes de lutte contre le chômage de fond (Freyssinet, 2006). Les années 1990 marquent, enfin, un nouveau cap avec un durcissement des positions et mesures concernant les chômeurs (Dubois, 2006) qui se traduit entre autres par une diminution progressive de la durée et du montant de leur indemnisation. (Daniel, Tuschszirer, 1999).

Ces orientations s'inscrivent dans la thématique de l' « activation », néologisme apparu depuis plusieurs années dans le vocabulaire des organisations internationales (CERC, 2005). Ainsi, selon l'OCDE, la politique d'activation vise la réduction des dépenses passives en faveur de l'emploi (indemnisation chômage) et l'augmentation des dépenses actives destinées au financement d'actions de réinsertion des chômeurs (formations, contrats aidés, « accompagnement », etc.). Par extension, on parle désormais couramment de l'activation des chômeurs. Activation et retour rapide en emploi représentent les objectifs prioritaires de la Stratégie Européenne de l'Emploi mise en place à partir de 1997. Le Traité d'Amsterdam, en juin 1997, accorde une place formelle aux questions de l'emploi et insiste sur la prévention du 
risque de chômage de longue durée. Ces orientations européennes ont eu des retombées visibles en France à travers l'instauration en 2001 du " Plan d'aide au retour à l'emploi » (PARE). Même si le mouvement était déjà amorcé, on peut dire que le PARE marque le point de départ de l'intensification de l'《 accompagnement » des chômeurs. Cette orientation a été encore dernièrement accentuée avec la mise en place du Suivi mensuel personnalisé (SMP). Depuis janvier 2006, les demandeurs d'emploi sont suivis individuellement par un conseiller référent qui, à partir de leur troisième mois d'inscription, les convoque tous les mois en entretien.

Déjà grandement répandue, la notion d'《accompagnement» imprègne désormais complètement le vocabulaire des institutions spécialisées dans l'aide à la recherche d'emploi. Elle offre une élasticité sémantique qui la rend socialement convenable. La dimension de contrôle (que laisse transparaître l'utilisation du terme "suivi » dans l'intitulé SMP) est gommée, sans être évacuée, tout en ennoblissant le rôle du conseiller chargé non pas d'aider, assister ou diriger un chômeur, mais de le rendre plus armé et plus actif dans sa recherche d'emploi :

«A la rigueur, je préfère accompagnement à suivi, parce que je trouve dans suivi, y a peutêtre plus une notion de contrôle que d'accompagnement, c'est comme ça que je le ressens personnellement. Quand on dit suivi mensuel personnalisé, y a plus un côté contrôle, je trouve. C'est vraiment : je suis à la trace la personne pour savoir où elle en est. (...) Mais je préfère accompagnement parce que, ouais, dans l'accompagnement, y a la notion de... on laisse de l'autonomie ... » (Conseiller référent, Anpe).

La connotation positive du terme d'accompagnement est actuellement d'autant plus importante aux yeux des conseillers que ces derniers sont confrontés aux contraintes de la logique d'activation, dont la principale conséquence est de renforcer leur mission de placement des chômeurs, y compris celui des moins « plaçables » ${ }^{7}$.

\section{4- L' « accompagnement » des chômeurs : une « dé-psychologisation » sous-tendue par des principes gestionnaires}

Si l'adoption de la logique d'activation des chômeurs ne modifie pas le contenu de la « boîte à outil » des conseillers (ils utilisent toujours les mêmes techniques et prodiguent toujours les mêmes conseils sur la présentation de soi du candidat lorsqu'il est en interaction avec un recruteur), elle conduit en revanche à une activation des conseillers eux-mêmes qui n'est pas sans conséquence sur leurs pratiques.

Tous les acteurs concernés par la recherche d'emploi sont en effet placés sous une forte pression temporelle : l'objectif est d'aller plus vite, d'accélérer le retour à l'emploi ou, dit autrement, de raccourcir la période de chômage. Les agents de l'ANPE doivent appliquer des mesures de plus en plus intenses : démarrage du suivi du demandeur d'emploi le plus tôt possible, rendez-vous obligatoires de plus en plus fréquents, contrôle de la réalité de la recherche d'emploi. Le conseiller doit tenir le rythme et " gérer » un portefeuille d'environ 120 demandeurs. Co-traitants et sous-traitants de l'ANPE n'échappent pas à cette logique : dans le cadre des prestations dont ils ont la charge, ils doivent également donner la priorité au retour rapide à l'emploi ${ }^{8}$.

\footnotetext{
${ }^{7}$ Terme indigène fréquemment utilisé entre autres dans les agences locales de l'Anpe.

${ }^{8}$ Par ailleurs, en vertu de la loi de Cohésion sociale de janvier 2005, l'UNEDIC est désormais autorisée à financer des opérateurs privés de placement (OPP) pour leur confier l'accompagnement de demandeurs
} 
Les conseillers se voient ainsi dans l'obligation de presser les chômeurs dans leurs démarches. Leurs principes d'intervention empruntés aux travailleurs sociaux s'avèrent alors peu compatibles avec ceux de l'activation. Ils disent même être amenés « à faire à la place » du chômeur pour gagner du temps, en appelant, par exemple, en leur nom un employeur. Ils sont ainsi contraints de renoncer à certains de leurs principes d'intervention auprès des chômeurs ; difficile désormais de «travailler leur demande» ou de compter sur leur implication personnelle :

"Réfléchir à ça [l'étape d'introspection], sur soi, dans la façon dont, nous, on a travaillé ces actions-là, si la personne n'est pas volontaire, on peut pas lui demander ce travail-là, parce que c'est quand même quelque chose d'assez impliquant, donc ça veut dire qu'il faut faire un autre travail avec elle. Et qui va être un travail qui, moi à mon sens, est beaucoup moins riche, parce que ça va être simplement faire des $C V$ et des lettres de motivation standardisés et être sur de l'opérationnel!' (Conseillère portant le titre de formatrice, salariée d'une association).

S'observe ainsi une tendance à la «dé-psychologisation» des pratiques ou modes d'intervention des conseillers auprès des chômeurs. Cette tendance est constamment renforcée par les dispositifs d'encadrement et d'évaluation de l'activité des conseillers, organisés autour de critères quantitatifs, se traduisant par une formalisation accentuée des tâches, et souvent dénoncés pour leur caractère bureaucratique. Il en est ainsi, par exemple, de la mise en place de logiciels d'aide au diagnostic des conseillers de l'ANPE qui évacuent les variables non mesurables et subjectives dans le "profilage » des chômeurs et leur orientation dans des parcours de recherche d'emploi pré-définis (Georges, 2006). Il en va de même pour les indicateurs « de pilotage » constitués par divers taux de « placement», de « sorties positives » [du chômage], de « non adhésion » [à une prestation], etc.

Cette évolution est également entretenue par les politiques de recrutement de conseillers par les responsables de structures d'aide à la recherche d'emploi. Ainsi, un directeur régional d'une association, spécialisée dans l'accompagnement des jeunes diplômés, déclare ne retenir aucune des candidatures de psychologues cliniciens. Une responsable de l'Anpe, qui participe à des jurys de recrutement de conseillers, insiste sur le fait que désormais résistance au stress temporel et connaissance du monde du travail sont des compétences très appréciées :

"Ce n'est pas: 'Ah, je suis là pour l'aider parce que le pauvre, il a plus de travail'. Les gens qui auraient cette vision là du métier, je pense qu'ils n'y trouveraient pas du tout leur compte, parce qu'aujourd'hui, il faut travailler vite, et c'est pas facile parce que vous avez des entretiens qui sont minutés, un entretien de suivi mensuel, c'est 20 mn!" (Animatrice d'équipe, Anpe).

\section{Conclusion}

Le champ du traitement social des chômeurs n'a pas été oublié par le processus de rationalisation qui d'après Max Weber (1965) va croissant dans tous les domaines de la vie. Au moins deux vagues successives de rationalisation se sont produites à environ vingt années

d'emplois indemnisés. Aussi, de nouveaux intervenants sont venus grossir les rangs des prestataires déjà présents sur le marché de l'accompagnement, et de ce fait renforcer la concurrence existante. 
d'intervalle. Dans un premier temps, s'est instaurée une normalisation de la recherche d'emploi des chômeurs. Les emplois de conseillers se sont multipliés et ces professionnels ont recouru à une approche psychologisante, notamment parce qu'ils disposaient de temps, la période de recherche d'emploi n'étant pas aussi accélérée qu'aujourd'hui.

A l'ère de l'« accompagnement », le retour rapide en emploi est devenu l'objectif prioritaire. Les conseillers sont soumis aux effets d'une rationalisation institutionnelle qui passe par l'élaboration de procédures détaillées, le recours à des logiciels et méthodes d'évaluation des dispositifs d'accompagnement. En suivant le constat que fait Charles Gadea (2003) à propos des cadres, on peut dire que les conseillers s'apparentent à des « rationalisateurs rationalisés ", à leur tour soumis à ce processus de rationalisation d'ordre non seulement formel, mais aussi idéologique puisqu'il s'inscrit dans une vision gestionnaire et managériale du monde (Dujarier, 2006), qui sous-tend les orientations des politiques de l'emploi. Dans un tel contexte, se produit une "dé-psychologisation» de l'aide à la recherche d'emploi. L'activation des chômeurs entraîne également celle des professionnels: de plus en plus soumis à des obligations de résultats, ils prennent une part plus active aux côtés du chômeur, jusqu'à parfois « faire à sa place » certaines démarches. D'une responsabilisation du chômeur, on passe à un partage des responsabilités entre l'intervenant et son interlocuteur. Ce rééquilibrage tend à réduire la " priorité de la norme d'intériorité » (Castel, 2003) qui pesait sur les seules victimes du chômage. Parallèlement, l'activité des « accompagnateurs » se resserre autour du placement dans l'emploi, quelles que soient les conditions de possibilités de remplir cette mission, et ce avec un moindre recours à des interprétations psychologisantes qui représentaient pour eux une échappatoire face aux contradictions de leur fonction.

sophie.divay@univ-paris1.fr

\section{Références bibliographiques}

Balmary Dominique, 2004, Rapport de l'instance de la politique de l'emploi et recours à des opérateurs externes, Paris, Commissariat au Plan, La Documentation française.

Beaud Stéphane, 1996, "Stage ou formation? Les enjeux d'un malentendu. Notes ethnographiques sur une mission locale de l'emploi », Travail et emploi, nº67.

Beauvois Jean-Léon, Le Poultier François, 1985, "Travail social et norme d'internalité », Connexions, $\mathrm{n}^{\circ} 46$.

Benarrosh Yolande, 2000, «Tri des chômeurs: le nécessaire consensus des acteurs de 1'emploi », Travail et emploi, $\mathrm{n}^{\circ} 81$.

Béraud Mathieu, 2006, "L'inemployabilité est-elle devenue une nouvelle pathologie mentale ? ", Les nouveaux cahiers du Grée, $\mathrm{n}^{\circ} 3$, septembre.

Castel Robert, 2003, L'insécurité sociale, Paris, Ed. du Seuil et La République des idées.

Castra Denis, 2003, L'insertion professionnelle des publics précaires, Paris, PUF.

CERC, 2005, Aider le retour à l'emploi, Rapport du Conseil, des revenus et de la cohésion sociale, Paris, la Documentation française.

Daniel Christine, Tuschszirer Carole, 1999, L'Etat face aux chômeurs. L'indemnisation du chômage de 1884 à nos jours, Paris, Flammarion.

Divay Sophie, 2001, «Chômage, malchance et traitement social », Ethnologie Française, n ${ }^{\circ} 1$, janvier - mars.

Divay Sophie, 2000, «L'aide à la recherche d'emploi : une activité de travail en voie de professionnalisation? », Travail et emploi, $\mathrm{n}^{\circ} 81$, janvier. 
Divay Sophie, 1999, L'aide à la recherche d'emploi. Des conseils pour sauver la face, Paris, l'Harmattan

Dubois Vincent, 2006, Le contrôle des demandeurs d'emploi, Paris, Rapport de recherche pour la DARES, ministère du travail.

Dujarier Marie-Anne, 2006, L'idéal au travail, Paris, PUF.

Frétigné Cédric, 2004, Une formation à l'emploi ?, Paris, L'Harmattan.

Freyssinet Jacques, 2006, L'émergence des politiques de l'emploi. (1945-1973), Paris, Centre d'Etudes de l'Emploi, Document de travail, $n^{\circ} 65$.

Gadea Charles, 2003, Les cadres en France. Une énigme sociologique, Paris, Belin.

Georges Nathalie, 2006, Le profilage : outil statistique et/ou de coordination?, Paris, Centre d'études de l'emploi, Document de travail, $\mathrm{n}^{\circ} 72$.

Goffman Erving, 1969, Le parler frais d'Erving Goffman, Paris, Les Editions de Minuit.

Ion Jacques, Ravon Bertrand, 2005, Les travailleurs sociaux, Paris, La Découverte.

Jellab Aziz, 1997, Le travail d'insertion en Mission locale, Paris, L'Harmattan.

Macé Eric, 1997, « Les contours de la médiation : institution, conciliation, conformation. A propos d'un dispositif de "médiation" de la RATP », Revue française des affaires sociales, $\mathrm{n}^{\circ} 2$.

Mauger Gérard, 2001, «Les politiques d'insertion. Une contribution paradoxale à la déstabilisation du marché du travail », Actes de la recherche en sciences sociales, ${ }^{\circ} 136-137$.

Orianne Jean-François, 2005, "Le traitement clinique du chômage », Journées d'études GRACC - GT19 de l'AISLF, "Y a-t-il psychologisation de l'intervention sociale? ” Lille, octobre.

Pochic Sophie, 2007, «Développer la recrutabilité. L'autre versant des transitions professionnelles », Cadres-CFDT, n $425-426$.

Vrancken Didier, Macquet Claude, 2006, Le travail sur soi. Vers une psychologisation de la société ?, Paris, Belin.

Weber Max, 1965 [1904], Essais sur la théorie de la science, Paris, Plon. 